\title{
A Software-Agnostic Agent-based Platform for Modelling Emerging Mobility Systems
}

\author{
Lampros Yfantis \\ University College London \\ London, United Kingdom \\ lampros.yfantis.17@ucl.ac.uk
}

\author{
Simon Stebbins \\ University College London \\ London, United Kingdom \\ s.stebbins@ucl.ac.uk
}

\author{
Ilias Gerostathopoulos \\ MOBY X Ltd \\ Limassol, Cyprus \\ i.gerostat@mobyx.co
}

\author{
Tamara Djukic \\ Aimsun SL \\ Barcelona, Spain \\ tamara.djukic@aimsun.com
}

\author{
Jordi Casas \\ Aimsun SL \\ Barcelona, Spain \\ jordi.casas@aimsun.com \\ David Garcia \\ Aimsun SL \\ Barcelona, Spain \\ david.garcia@aimsun.com
}

\author{
Maria Kamargianni \\ University College London \\ London, United Kingdom \\ m.kamargianni@ucl.ac.uk
}

\author{
Manos Chaniotakis \\ University College London \\ London, United Kingdom \\ m.chaniotakis@ucl.ac.uk
}

\begin{abstract}
Due to the rapidly accelerated innovation cycle in transport and the emergence of new mobility concepts and technologies, public authorities, policy makers, and transport planners are currently in need of the tools for sustainable spatial and transport planning in the new mobility era. In this paper, a new modular, software-agnostic and activity-based spatial and transport planning platform is designed, i.e, the HARMONY Model Suite, that facilitates a novel integration of new and existing spatial and transport modelling tools. The paper focuses on describing the architecture of the platform and its passenger mobility simulation framework, which integrates -in an interoperable manner- activity-based models, mobility service management, and traffic simulation tools for evaluating new mobility system dynamics. The service management controllers for new mobility concepts are discussed in more detail with regards to their functionality and applicability.

Index Terms-simulation platform, agent-based, softwareagnostic, new mobility services, operations
\end{abstract}

\section{INTRODUCTION}

Metropolitan areas' densely populated urban cores and sprawling suburban areas are responsible for over two thirds of greenhouse gas emissions and energy consumption [1]. As such, the need to place them and their regional spatial and transport planning apparatus at the very heart of any attempt to address increasing traffic congestion and the COP22 climate goals is imperative. The rise of the sharing economy along with significant advancements in vehicle automation, artificial intelligence and Information and Communication Technology (ICT) systems have led to the emergence of disruptive passenger and freight transport concepts such as on-demand and shared mobility services, autonomous mobility, urban air mobility and first-/last-mile shipping solutions [2]-[4]. Such concepts are receiving industrial and academic attention, due to their significant potential regarding sustainable multimodal cities but also, due to the uncertainty associated with their implementations and the possible negative externalities they might induce.

978-1-7281-8995-6/21/\$31.00 @2021 IEEE
With the rapidly accelerated innovation cycle, public authorities, policy makers and transport planners currently lack the tools for multi-scale spatial and transport planning in the new mobility era. Consistent analysis of new policies and interventions for new mobility concepts and technologies require feasibility assessments based on extensive experimentation and multiple replications of simulation scenarios. The HARMONY H2020 project aims to address this need with the development of the HARMONY Model Suite (MS) - a new modular, software-agnostic and activity-based spatial and transport planning platform that facilitates modelling of spatial organization and new dynamics of multimodal freight and passenger transport systems. The platform is software-agnostic in the sense that it does not prescribe the use of a particular software component, technology or language for its components. From a passenger transport modelling perspective, the main goal of the HARMONY MS is to bring together within one modular platform newly developed but also existing tools that enable simulating in detail the behavioral and operational dynamics of emerging multimodal transport systems.

Although integrated agent-based and multimodal demand and supply models that consider new mobility system dynamics exist in the pertinent literature [5], [6], to the best of the authors' knowledge, such a software-agnostic transport simulation platform that consistently brings together independent activity-based demand and traffic simulation tools for new mobility system models has not been investigated or proposed. As such, the objective of this paper is twofold. First, it provides an initial description of the envisioned HARMONY MS, focusing on the design of its flexibly integrated multiscale passenger mobility simulation framework. Secondly, it presents the high-level architecture of novel new mobility service management controllers which aim to emulate dynamic operations of innovative mobility services and enhance existing within-day traffic simulation models with new mobility dimensions in an interoperable manner.

The remainder of the paper is structured as follows. Section II motivates HARMONY MS, while Section III overviews 
its architecture. Section IV describes the passenger mobility simulation framework of HARMONY MS, while Section V elaborates on the design of its service management controllers. Finally, Section VI concludes and discusses future steps.

\section{Motivation for Harmony Model Suite}

The overarching goal of HARMONY is to develop a virtual "test bed" that transport planners, decision makers and service operators can utilise to represent transport systems with new mobility services (on-demand, shared, MaaS) and technologies (autonomous and/or electric vehicles). Similar to other transport policies and interventions (e.g., low-emission zones and new public transport systems), the introduction of a new city-wide or metropolitan-wide mobility system (e.g., MaaS or autonomous on-demand mobility) may have a multifaceted and multi-scale impact including changes in land use and travel patterns, transport network efficiency and the environment. Therefore, the evaluation of new mobility concepts entails the utilisation of multi-dimensional frameworks that capture i) infrastructural, behavioural and operational elements of new transport systems and ii) the propagating effects of such interventions among interrelated spatial organization, people's behaviour and transport networks.

At the same time, Planning authorities are also faced with the challenge of updating their transport models with more sophisticated demand representations (activity-based) and enhanced networks that capture new mobility systems. Widelyused traditional transport modelling software (e.g., Aimsun Next, PTV VISUM/VISSIM, SUMO, Paramics, Saturn) have, in most cases, certain limitations with regards to i) more realistic transport demand representations at the agent-level and ii) representing organizational and operational particularities of new disruptive mobility systems. However, in many cases planning authorities refrain from transitioning to new modelling tools which would require significant cost and time resources for re-training existing or hiring new personnel. The HARMONY project aims to facilitate and enable the transition from trip-based to agent-based demand models that capture within-day operational and networks dynamics in the presence of new transport concepts. In fact, the design of the envisaged HARMONY MS is driven by the need to interoperably, flexibly and consistently integrate in one multi-scale platform existing transport simulation tools with new modelling components that enhance the range of their functionality and applicability.

\section{OVERVIEW OF HARMONY MODEL SUITE}

In light of the above, the HARMONY MS is composed of three loosely coupled levels which capture urban and/or transport system dynamics in different time scales (Fig. 1):

Strategic Modelling Level: it is a model system consisting of regional economic, demographic forecasting, land-use, spatial freight interaction, population synthesis and long-term mobility choice models operating on a long-term horizon (e.g., year-to-year) [7]; this level is mainly responsible for predicting (i) synthetic household and firm populations and their locations, (ii) land-uses (locations for different types of activities such as employment, housing, and education), (iii) aggregate commodity flows between employment sectors, and (iv) long-term mobility choices of traveller agents, including teleworking decisions, car-ownership (vehicle stock) and subscriptions to different mobility services (e.g. MaaS).

Tactical Modelling Level: this level encompasses fully agent-based daily passenger and freight demand simulators, as well as a day-to-day learning sub-module that updates traveller agent's perceptions (by experience) of network performance [8], [9]. The activity-based passenger demand modelling framework considers individuals, households, and the interaction of individuals within the same household and predicts their activity choices throughout a day and their corresponding travel decisions (mode choices, departure times, etc.). The multi-agent freight demand simulator simulates individual firms and shipments and the logistic decision-making choices of freight stakeholders. The output from both submodels involves disaggregated demand in the form of agents' daily activity schedules and disaggregated demand in the form of freight vehicle tours/trips (i.e., trucks, vans, freight bikes, etc.).

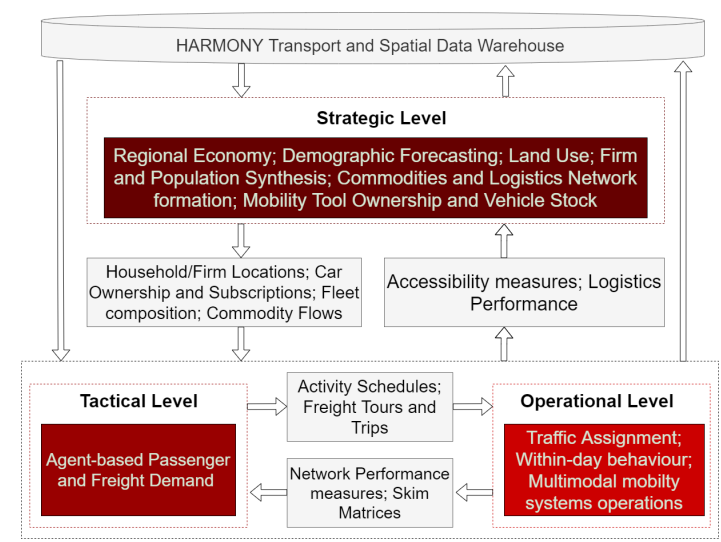

Fig. 1. The HARMONY Model Suite conceptual architecture.

Operational Modelling Level: this level models transport supply and demand interactions at high granularity (e.g. second to second, minute to minute). It is designed as a hybrid multimodal passenger and freight traffic simulation system that utilises as input both activity-based demand data in the form of schedules and tours. It is responsible for (i) capturing travellers' dynamic schedule re-evaluation and routing/re-routing choices, (ii) loading passenger and freight demand into the transport network, and (iii) emulating realistically disruptive new mobility and freight services and concepts. The output of the Operational level includes mainly network and service performance, energy and emission indicators.

To enable modular design, each distinct capability of the HARMONY MS is wrapped into an independently developed and deployed component (e.g. traffic simulator, service controller). The modular design of the platform's architecture dictates that each level, as well as each component in a level, can run in isolation assuming that necessary exogenous inputs 
are provided. At the same time, all three levels can also run sequentially through consistent input/output interactions. A common data schema and database, i.e, a Transport and Spatial Data Warehouse (TSDW) facilitates the consistent storage and communication of information and data across simulation runs. The run-time communication between components within different HARMONY MS levels is facilitated via Kafka and Google's Remote Procedure Call-based (gRPC) Application Programming Interfaces (APIs). Different scenarios allow end users to specify which components should be invoked, at which order, and which analyses should be performed during and after the simulation runs. Both the metadata of scenarios, their inputs, and the simulation outputs are stored in the HARMONY TSDW for post-processing analysis and scenario evaluation and tracking. Finally, a web-based Graphical User Interface enables end users to specify and run different scenarios via dedicated scenario templates that define the required inputs with regards to model entry points, database input/outputs and model configurations. Scenarios for interventions are supported in the backend via workflows, which can either be rigid or adaptive (as illustrated in Fig. ??), i.e. they can dynamically decide which component of the workflow should be selected to run or which component(s) should be skipped, based on the sofar calculated performance indicators and available variation points provided by the workflow designers.

\section{Passenger Mobility Simulation Framework}

Among its various functionalities, the HARMONY MS aims at enabling the modelling and evaluation of multimodal emerging passenger transport systems via an integrated multi-scale demand and supply modelling framework, i.e., the Passenger Mobility Simulator. Its high-level architecture is depicted in Fig. 2. The design of the Strategic Level of the Passenger Mobility Simulator incorporates two main modules, i.e., a Population Synthesis module and a Long-term Mobility Choice model system. The Population Synthesis module [10] utilizes total control variables from either exogenous sources (e.g., census and travel surveys) or model-predicted data (outputs of demographic forecasting and land-use models) towards generating an initial synthetic population of households and individuals (traveler agents) along with their locations and basic socio-demographic attributes. Long-term mobility choice models [8] can then be utilized to enhance the initial synthetic households and individuals with predicted socio-economic attributes that may not be available in census or national survey data, including vehicle ownership, subscriptions to MaaS and teleworking decisions.

The synthetic population along with exogenously accessed or model-predicted land use and skim matrices feed the Tactical Level, which is responsible for generating high-level habitual activity schedules/plans for each agent in the population. The Tactical Level consists of a daily activity-based passenger travel demand simulator [8] that utilizes generationallocation and scheduling models to predict such daily activity schedules in the form of tours and trips performed by agents to participate in specific activities (e.g., work, education, leisure, etc.). The final output of the simulator includes habitual plans for each agent in the synthetic population, including high-level information such as activity duration, activity locations, trip departure times and travel modes for each trip.

Transport demand in the form of daily activity schedules along with the synthetic population data are fed to the Operational Level, which is mainly responsible for the execution of these schedules. The Operational Level is envisaged as an integrated agent-based model system that simulates withinday demand-supply interactions at a high level of granularity (e.g., seconds or minutes). It enables the representation of different agents, such as travelers, fleet vehicles/drivers and new mobility and MaaS service providers, as well their dynamic within-day interactions and actions. The main facilitator of this functionality is an Agent Controller System (ACS) that includes an Agent Schedule Manager, a Mobility Service Controller, and a MaaS Controller. The Agent Schedule Manager enables the representation of agents with their dynamic withinday behavior (e.g., schedule re-evaluation) and the subsequent translation of schedules into point-to-point trips to be simulated by the traffic simulator, while the Mobility Service and MaaS Controllers emulate new multimodal mobility service and fleet operations (e.g., MaaS, autonomous mobility, ondemand, and shared services).

As already mentioned, an important design requirement of the HARMONY MS is to enable the utilization and integration of existing traffic simulation tools. To this end and based on the scope of the HARMONY project's pilots, the Operational Level design currently foresees two different types of integration with two popular transport modeling software platforms, i.e., PTV VISUM and Aimsun Next. For the former, which has been selected based on its widespread use in the EU for planning purposes, the integration of demand in the form of activity schedules into the network model relies on their apriori translation into Origin-Destination (OD) matrices. It should be pointed out that this particular strategy has been rendered necessary due to the design nature of the VISUM model, however it limits the exploitation of the Agent Controller System's full functionality. For the latter, the Aimsun Next model is a mesoscopic and microscopic traffic simulator that offers APIs enabling online communication with external modules at a trip level. This further allows the Agent Controller System to publish agents' trips and receive events at runtime via a gRPC-based data adapters. Network and service performance data computed by the network models are fed back to the Tactical Level and converted into skim matrices via a day-to-day learning module that updates agent's perceptions (by experience) of network performance; an essential process for calibration of model convergence processes, as indicated in [5]. Finally, detailed link-level speed and vehicle type data are utilized by an Energy and Emission model [11] to subsequently estimate air pollutants and energy consumption. The integration of the ACS with Aimsun Next is facilitated via an extension of the Ride API, a gRPC-API that allows the simulation of agents' (travellers, fleet vehicles) trips computed by the Agent Schedule Manager and the service controllers. 


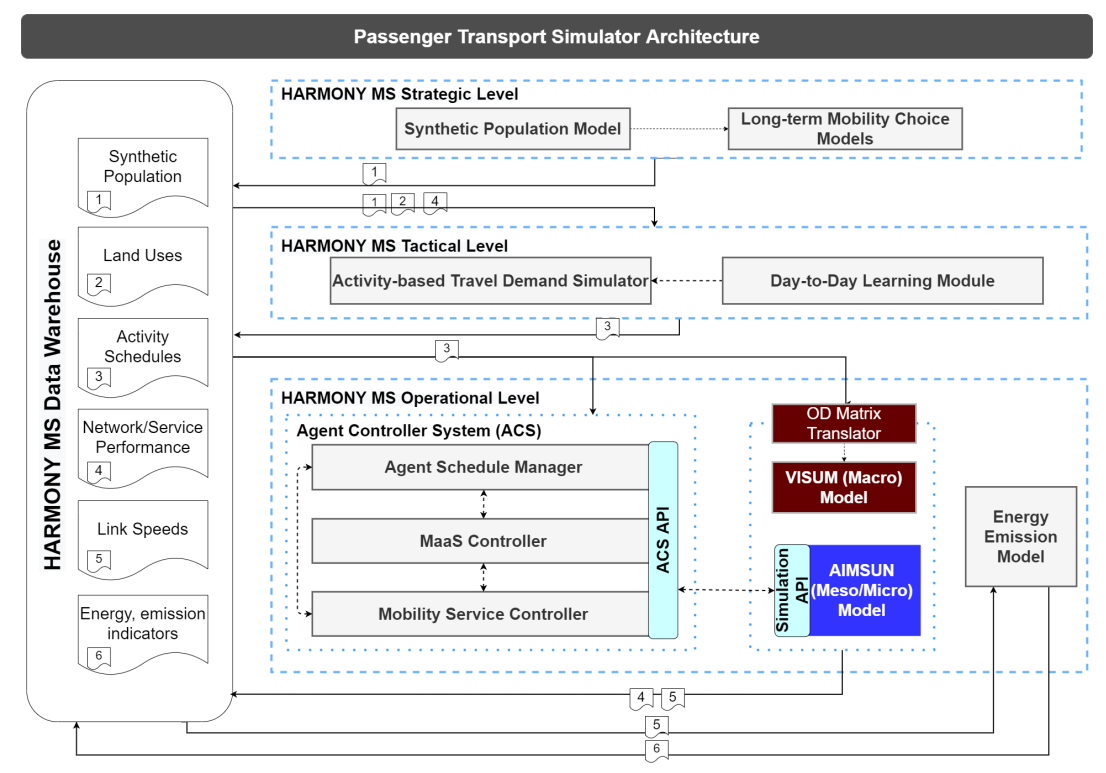

Fig. 2. Passenger Mobility Simulation framework.

Trips may consist of either one or more modes (e.g., walk, ride hail, PT, private car). The RideAPI notifies the ACS when an event takes place in the simulation (e.g., trip and trip leg initiation and completion, increasing congestion) which might cause schedule or fleet trip re-evaluation. Furthermore, the simulation provides a path calculation functionality that is used by the ACS for determining fleet vehicle or traveller agent routes when and if needed. This functionality relies on path costs that are estimated by (i) free flow conditions, (ii) simulated data using the Dynamic Traffic Assignment, (iii) historical travel time data.

\section{Controllers for New Mobility Services}

he HARMONY MS includes dedicated controllers that emulate required service platform operations and their dynamic interactions with their environment. The design and reference architecture of the envisioned controllers are driven by four main goals:

- Enhancing the ability of existing traffic simulators to consider a range of new on-demand mobility services, concepts and technologies;

- Realistically representing complex within-day operational dynamics of multimodal transport ecosystems composed of competing and/or collaborative services and providers;

- Producing a framework that can accommodate a flexible integration with different demand (activity-based) and simulation-based traffic assignment models;

- Enabling the consistent evaluation of different service designs and operational strategies on urban transport demand and supply.

To achieve these goals, a flexible modular and agent-based controller design approach is adopted. The agent-based design relies on the object-oriented programming (OOP) paradigm which facilitates the representation of each mobility service or MaaS platform and their assets (stations, fleet, products) as objects in the simulation. The modularity of the controllers' design allows end-users to switch on and off certain controllers and controller functionalities or even override existing functions through the scenario templates discussed in Section 2. At the same time, the flexibility in the communication between different controller types and with other components of the Operational Level (e.g. Agent Schedule Manager, Traffic Simulator) is enabled via a gRPC-based interfacing system. Below, the architecture and the functionalities of the envisioned/proposed controllers are described in more detail.

\section{A. Mobility Service Controller}

The Mobility Service Controller uses input demand from the Agent Schedule Manager in the form of trip requests from A to B for a specific departure time with a specific mode. For each request the main goal of the controller is to assign an available fleet vehicle to serve the request. Several assignment functions will be developed depending on the service type (single ride, shared ride, van pooling, humandriven or autonomous vehicle), the modes that are supported by the controller and the context of application. Besides the assignment functionality, optimal fleet relocation and dynamic pricing features will be also developed.

The existing operational prototype of the Mobility Service Controller includes the representation of an on-demand ridehailing service offering single rides from $\mathrm{A}$ to $\mathrm{B}$. For that example, a typical sequence of interactions once a trip request is received is as follows. A passenger agent retrieves a controller object from the simulation experiment and sends a trip request message to the controller. The controller queries the simulation adapter to find suitable vehicles that can fulfil the request. For example, nearby vehicles that can reach the passenger in the least amount of time may be returned. The 
controller identifies optimal vehicle-request matches and offers the trip to the vehicles in order of priority. If a vehicle (driver) rejects the request, the next vehicle is offered the trip. If a trip is accepted by a driver, the passenger is notified that a trip is available. If no vehicle was found to fulfill the trip, the passenger waits until a pre-defined threshold and if no vehicle is assigned he/she leaves the system. The passenger evaluates whether to accept or reject the trip. The controller then confirms the trip with the driver agent and the computed fleet vehicle trip is sent as a trip execution command to the simulator through the simulation adapter. It should be noted that an adapter component is required for each simulator to "translate" generic trip execution commands from the mobility service controller in a manner that is understood by the API of each specific simulator.

\section{B. MaaS Controller}

The MaaS Controller represents the platform (front-end/app, back-end) of a MaaS operator (e.g., Whim, Citymapper) and emulates operations required for dynamically and optimally integrating, allocating/distributing and suggesting resources (mobility service alternatives) to traveler agents that are subscribed to the particular MaaS service and have selected a MaaS mode (Tactical Level input) for a trip. The design of the MaaS Controller is, in fact, based on the conceptual framework originally proposed in [12] as an extension of the SimMobility platform [5] and shall enable the following functionalities:

- Analysis of MaaS scenarios and extraction of outputs such as daily MaaS trips per mode and service provider, deviations between performance of suggested and realised MaaS trips, MaaS user satisfaction indicators, service provider performance estimates;

- Design of one or more MaaS operators for simulating potential competitive ecosystems with several MaaS and mobility service providers;

- Design of MaaS products, MaaS accounts and contractual agreements between MaaS and mobility service providers;

- Emulation of realistic agent interactions (travellers, MaaS providers, Mobility Service providers) along with the ability to communicate service performance data in different formats and detail;

- Integration of different strategic and dynamic operational strategies, such as pricing, dynamic trip planning under different settings, predictive and learning functionalities.

The high-level architecture of the MaaS Controller and the main offline and online input/output interactions with other Operational level's components are illustrated at Fig. 3. The HARMONY MS's data warehouse and configuration files dictate the MaaS simulation scenarios and experiments, including offline generated inputs such as modes offered by MaaS Controller(s), whether MaaS controllers are activated, and details on the service design (products, subscription penetration rates, contracts, strategies). The activity-based Passenger Demand simulator (Tactical Level) is responsible for generating the MaaS demand based on traveller agents' subscription choices and account status. The demand takes the form of daily activity schedules for synthetic traveller agents that are subscribed to a MaaS service and have chosen a mode for their trip(s) through a MaaS provider. Such schedules (for both MaaaS and non-MaaS modes) are stored in the database and constitute the main input of the HARMONY MS Operational Level for dynamic within-day simulations.

At the Operational Level, the Initialiser module of the MaaS Controller is responsible for instantiating the required structures of the controller (e.g., MaaS network model) and creating connections with the controller's user accounts based on input data and configurations. The MaaS Controller is comprised of two main modules that manage MaaS demandand supply-related operations at simulation run time and interact dynamically with other components of the Operational level. The MaaS Demand Manager receives the MaaS demand in the form of trips requests from $\mathrm{A}$ to $\mathrm{B}$. The requests are generated from the Agent Schedule Manager and emerge from translating the activity schedules into actual trips with specific origin, destination, departure time and mode. For each trip request, the MaaS Demand Manager generates optimal multimodal travel menus including various mode-route options based on (i) pre-configured resource allocation strategies (e.g., personalization, revenue maximization, sustainability goals), (ii) the status of a user MaaS accounts (remaining capacity), and (iii) contractual agreements with mobilty service providers. Menus are dynamically-generated choice sets which traveller agent may choose from. Menus are updated with real-time trip-specific information from service controllers are presented to the traveller agent who chooses an option. If the agent has chosen an alternative from the menu, the choice is communicated to the MaaS Controller. At the same time, if the chosen MaaS trip includes a service offered by a Mobility Service Controller, the booking process is initiated. The MaaS Controller makes a trip request to the Mobility Service Controller on behalf of the agent and once the booking is verified, i.e., a vehicle has been assigned, confirmations are sent to the MaaS Controller and subsequently to the traveller for trip initiation. Upon trip completion, the agent's account is updated to reflect that a trip with a specific mode was completed.

\section{CONCLUSIONS AND Future WORK}

The focus of this paper is to provide an initial description of the overall envisioned architecture of new platform that facilitates agent-based passenger mobility simulations for emerging multimodal transport systems. While some of its components have been designed, ongoing research activities are being conducted towards its completion including extensive data collection for network model updates and demand estimation. The ultimate goal is to develop the proposed framework and apply it in the Oxfordshire County for evaluating the impact of MaaS and autonomous Demand Responsive Transit services. 


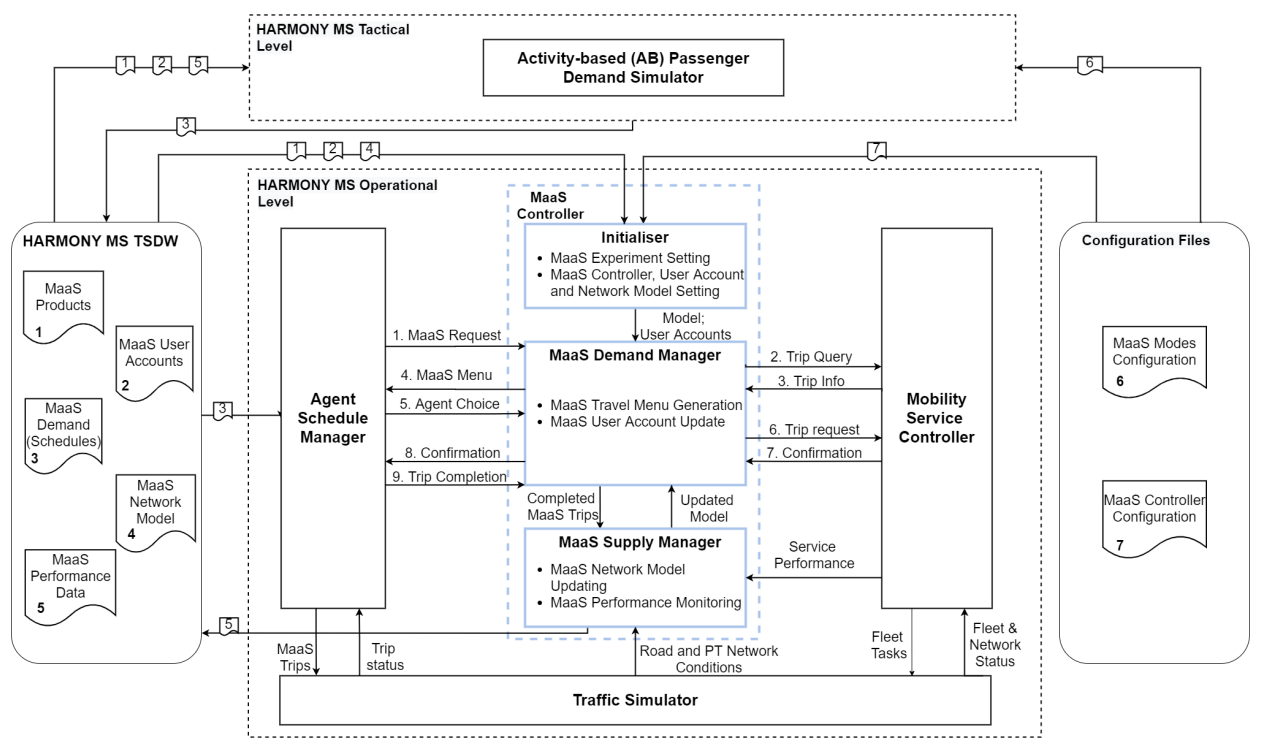

Fig. 3. MaaS Controller high-level architecture.

\section{ACKNOWLEDGMENT}

The research reported in this paper has been supported by European Union's Horizon 2020 research and innovation programme under Grant Agreement No 815269, project HARMONY.

\section{REFERENCES}

[1] World Bank, "The World Bank Annual Report 2016," Online; Available at https://openknowledge.worldbank.org/handle/10986/24985, Washington, DC., 2016.

[2] S. A. Shaheen, A. Bansal, N. Chan, and A. Cohen, "Mobility and the sharing economy: industry developments and early understanding of impacts," Low Carbon Mobility for Future Cities: Principles and applications, pp. 213-240, Feb. 2017, publisher: IET Digital Library.

[3] S. Narayanan, E. Chaniotakis, and C. Antoniou, "Shared autonomous vehicle services: A comprehensive review," Transportation Research Part C: Emerging Technologies, vol. 111, pp. 255-293, 2020.

[4] C. Al Haddad, E. Chaniotakis, A. Straubinger, K. Plötner, and C. Antoniou, "Factors affecting the adoption and use of urban air mobility," Transportation research part A: policy and practice, vol. 132, pp. 696$712,2020$.

[5] M. Adnan, F. C. Pereira, C. M. Lima Azevedo, K. Basak, M. Lovric, S. Raveau, Y. Zhu, J. Ferreira, C. Zegras, and M. Ben-Akiva, "SimMobility: a multiscale integrated agent-based simulation platform," Jan. 2016.

[6] A. Horni, K. Nagel, and K. W. Axhausen, The multi-agent transport simulation MATSim. Ubiquity Press, 2016.

[7] M. Batty, T. Evans, and F. Lopane, "D4.1 The HARMONY Strategic Simulator sub-models - Initial version," 2020, HARMONY project report.

[8] I. Tsouros, D. Pappelis, F. Song, and F. Fermi, "D5.1-Daily activity and travel patterns and dynamic demand-shift models," 2021, HARMONY project report.

[9] I. Kourounioti, M. De Bok, L. Tavasszy, and S. Thoen, "D6.1-The HARMONY Shipment synthesizer module -Initial version,” 2020, HARMONY project report.

[10] X. Ye, K. Konduri, R. M. Pendyala, B. Sana, and P. Waddell, "A methodology to match distributions of both household and person attributes in the generation of synthetic populations," in 88th Annual Meeting of the Transportation Research Board, Washington, DC, 2009.

[11] L. Dray, Y. Matsinos, and A. Tsaligopoulos, "D7.4 Energy, Emissions and Noise Models -Initial Version," 2021, HARMONY project report.
[12] M. Kamargianni, L. Yfantis, J. Muscat, C. M. L. Azevedo, and M. BenAkiva, "Incorporating the mobility as a service concept into transport modelling and simulation frameworks," 2019. 